九州大学学術情報リポジトリ

Kyushu University Institutional Repository

\title{
ON A METHOD TO EXTRACT RULES FROM A TABLE WITH NON-DETERMINISTIC INFORMATION: A ROUGH SETS BASED APPROACH
}

Sakai, Hiroshi

Department of Computer Engineering, Kyushu Institute of Technology

https://doi.org/10.5109/13506

出版情報: Bulletin of informatics and cybernetics. 34 (1), pp.13-28, 2002-10. Research Association of Statistical Sciences

バージョン：

権利関係 : 


\title{
ON A METHOD TO EXTRACT RULES FROM A TABLE WITH NON-DETERMINISTIC INFORMATION: A ROUGH SETS BASED APPROACH
}

\author{
By
}

\author{
Hiroshi SAKaI*
}

\begin{abstract}
Rough sets theory is now becoming a mathematical foundation of soft computing. This theory makes use of equivalence relations defined for each set of attributes in any table, and applies the concept like definability of a set, dependency among attributes, reduction of data, rule extraction, etc., to data analysis.

In this paper, a problem of knowledge discovering in the form of rules from any table with non-deterministic information is discussed. At first, the rough sets based concept including rule extraction is surveyed, and this concept is extended to new one related to non-deterministic information. Then, a framework of rule extraction from tables with non-deterministic information is proposed, and some algorithms for handling such new concept are presented. Also implemented programs and a real execution of these programs are shown.
\end{abstract}

Key Words and Phrases: Rule extraction, Rough sets, Non-deterministic information, Data dependency, Possible equivalence relation.

\section{Introduction}

Rough sets theory is seen as a mathematical foundation of soft computing. The theory covers important areas of research in AI such as knowledge, imprecision, vagueness, learning and induction (Pawlak, 1991;1996; Orlowska and Pawlak, 1984; Nakamura et al., 1996). It has been applied to machine learning and knowledge discovery by Polkowski and Skowron (1998a;1998b), Grzymala-Busse (1997), Ras and Joshi (1997), Tsumoto (2000) and Zhong et al. (1998).

Rough sets theory usually handles a table with deterministic information which we call DIS(Deterministic Information System). Recently for handling incomplete information like null values (Codd, 1970), unknown values (Lipski, 1981; Grzymala-Busse, 1991; Kryszkiewicz, 1998;1999; Sakai, 1998), etc., rough sets in a table with non-deterministic information, which we call NIS(Non-deterministic Information System), attract interest of researchers.

Already some important work on NISs has been conducted, but most work has focused on logic, in particular, an axiomatization of logic in NISs (Orlowska and Pawlak, 1984; Lipski, 1981). There is only few work that deals with real data analysis in NISs. For example, Lipski (1981) showed modal question-answering systems

\footnotetext{
* Department of Computer Engineering, Kyushu Institute of Technology, Tobata Kitakyushu 804 Japan. tel +81-93-884-3258 sakai@comp.kyutech.ac.jp
} 
besides the axiomatization of logic. Grzymala-Busse $(1991 ; 1997 ; 1998)$ surveyed the unknown attribute values, and studied the learning from examples with unknown attribute values. Kryszkiewicz $(1998 ; 1999)$ discussed the theoretical aspect of rules in incomplete information systems. These are the most important work related to NISs.

The research of rough sets based data analysis in NISs has still been untouched. Rough sets based data analysis in NISs could also be a mathematical foundation of knowledge discovery and data mining from incomplete information. In such a situation, we are now investigating rough sets based algorithms for data analysis, namely algorithms using equivalence relations in $D I S s$ and algorithms using possible equivalence relations in NISs. In (Sakai and Okuma, 1999; Sakai, 2001b), a manipulation of possible equivalence relations in NISs was studied. This work proposed a method to obtain all possible equivalence relations in any NIS. A new data dependency among attributes in NISs and algorithms for checking this dependency were also proposed (Sakai and Okuma, 2000; Sakai, 2001a). Algorithms using possible equivalence relations drastically reduced the execution time for checking this data dependency.

In this paper, the framework of rule extraction from $D I S s$ is extended to the framework of rule extraction from NISs. In every $N I S$, a rule is defined by a formula satisfying some kinds of constraints. Six groups of rules are introduced into NISs as a constraint, and the proposed dependency in $N I S s$ is applied as another constraint to formulas. The problem is reduced to pick up formulas satisfying constraints, and some effective algorithms are needed. For this problem, we applied algorithms using possible equivalence relations, and realized a tool to obtain rules from NISs. This framework follows the framework of rule extraction from DISs. Thus, it is possible to extract rules not only from $D I S s$ but also from NISs.

\section{A Survey of Rough Sets Theory}

This section surveys basic concept in rough sets theory.

\subsection{Some Definitions in Deterministic Information Systems}

A Deterministic Information System $(D I S)$ is a quadruplet $\left(O B, A T,\left\{V A L_{a} \mid a \in\right.\right.$ $A T\}, f)$, where $O B$ is a finite set whose elements are called objects, $A T$ is a finite set whose elements are called attributes, $V A L_{a}$ is a finite set whose elements are called attribute values and $f$ is such a mapping that $f: O B \times A T \rightarrow \cup_{a \in A T} V A L_{a}$ which is called a classification function. If $f(x, a)=f(y, a)$ for every $a \in A T R \subset A T$, we see there is a relation between $x$ and $y$ for $A T R$. This relation is an equivalence relation over $O B . e q(A T R)$ denotes this equivalence relation, and $[x]_{A T R}$ denotes an equivalence class with $x$. In this case either $[x]_{A T R}=[y]_{A T R}$ or $[x]_{A T R} \cap[y]_{A T R}=\emptyset$ holds for any $x, y \in O B$. Furthermore, $\cup_{x \in O B}[x]_{A T R}=O B$ also holds. If a set $X \subset O B$ is the union of some equivalence classes, we say $X$ is definable (for $A T R$ ) in $D I S$. Otherwise we say $X$ is rough.

Let us consider two sets $C O N \subset A T$ which we call condition attributes and $D E C \subset$ $A T$ which we call decision attributes. Two distinct objects $x, y \in O B$ are consistent (between CON and DEC), if $f(x, a)=f(y, a)$ for every $a \in C O N$ implies $f(x, a)=f(y, a)$ for every $a \in D E C$. A DIS is consistent (between $C O N$ and $D E C$ ), if every pair of objects is consistent. In this case, we may say DEC depends totally on CON.

Now, let us show an important proposition connecting dependencies with equiv- 
alence relations. Let $e q_{1}$ and $e q_{2}$ be two equivalence relations over $O B$. A formula $e q_{1} \subset e q_{2}$ means that for every equivalence class $L \in e q_{1}$ there exists such an equivalence class $M \in e q_{2}$ that $L \subset M$.

Proposition 2.1. (Pawlak, 1982;1991) For any DIS, condition attributes CON and decision attributes $D E C, 1$ and 2 in the following are equivalent.

1. DEC depends totally on CON.

2. $e q(C O N) \subset e q(D E C)$.

Positive region $P O S_{C O N}(D E C)=\cup\{L \in e q(C O N) \mid$ there exists such $M \in e q(D E C)$ that $L \subset M\}$ is applied to characterize dependencies. Each degree is measured by a ratio $\left|P O S_{C O N}(D E C)\right| /|O B|$, and this ratio is called the degree of dependency from $C O N$ to $D E C$. The degree of dependency is 1 if and only if $D E C$ depends totally on $C O N$.

\subsection{Rules in Deterministic Information Systems}

Let us survey important work for extracting rules in $D I S s$. For any $D I S$, let $C O N$ and $D E C$ be condition and decision attributes, respectively. For any $x \in O B$, a function $d_{x}: C O N \cup D E C \rightarrow \cup_{a \in C O N \cup D E C} V A L_{a}$ such that $d_{x}(a)=f(x, a)$ is introduced in DISs (Pawlak, 1991). $d_{x} \mid C O N$ denotes a formula $\wedge_{a \in C O N}[a, f(x, a)]$, and $d_{x} \mid D E C$ denotes a formula $\wedge_{a \in D E C}[a, f(x, a)]$. The formula $[a, f(x, a)]$ is called a descriptor, and it means that $f(x, a)$ is the value of the attribute $a$. Generally, a rule is defined by an implication $d_{x}\left|C O N \Rightarrow d_{x}\right| D E C$ satisfying some kinds of constraints.

The most familiar constraint is a dependency from $C O N$ to $D E C$. If the degree of dependency is more than a threshold value, each implication in DIS is a rule (Pawlak, 1991). Some criteria are applied to each implication as other constraint. Three criteria in the following are applied to each implication from $x$ (Tsumoto, 2000).

$\operatorname{support}(x)=\mid\left\{y \in O B\left|d_{y}\right| C O N=d_{x} \mid C O N\right.$ and $\left.d_{y}\left|D E C=d_{x}\right| D E C\right\}|/| O B \mid$, $\operatorname{accuracy}(x)=\left|[x]_{C O N} \cap[x]_{D E C}\right| /\left|[x]_{C O N}\right|$, $\operatorname{coverage}(x)=\left|[x]_{C O N} \cap[x]_{D E C}\right| /\left|[x]_{D E C}\right|$.

If each criterion value of an implication is more than each threshold value respectively, this implication is a rule.

Example 2.2. Let us consider a $D I S$ in Table 1.

\begin{tabular}{|c|c|c|c|}
\hline$O B$ & $A$ & $B$ & $C$ \\
\hline 1 & 2 & 2 & 1 \\
\hline 2 & 1 & 2 & 2 \\
\hline 3 & 2 & 2 & 1 \\
\hline 4 & 1 & 3 & 3 \\
\hline
\end{tabular}

Table 1: A deterministic information system

Here, $e q(\{A\})=\{\{1,3\},\{2,4\}\}$ and $e q(\{C\})=\{\{1,3\},\{2\},\{4\}\}$ hold. A set $\{1,2,3,4\}$ is definable for $\{A\}$, because this set is equal to $\{1,3\} \cup\{2,4\}$. However, a set $\{1,2,3\}$ is rough for $\{A\}$. Both sets are definable for attributes $\{C\}$. Since $e q(\{C\}) \subset e q(\{A\})$, $\{A\}$ depends totally on $\{C\}$, and every implication $d_{x}\left|\{C\} \Rightarrow d_{x}\right|\{A\}(x \in O B)$ is seen 
as a rule. This dependency generates rules in the following.

$\mathrm{d}_{1}\left|\{C\} \Rightarrow \mathrm{d}_{1}\right|\{A\} \quad\left(=\mathrm{d}_{3}\left|\{C\} \Rightarrow \mathrm{d}_{3}\right|\{A\}\right):[\mathrm{C}, 1] \Rightarrow[\mathrm{A}, 2]$,

$\mathrm{d}_{2}\left|\{C\} \Rightarrow \mathrm{d}_{2}\right|\{A\}:[\mathrm{C}, 2] \Rightarrow[\mathrm{A}, 1], \mathrm{d}_{4}\left|\{C\} \Rightarrow \mathrm{d}_{4}\right|\{A\}:[\mathrm{C}, 3] \Rightarrow[\mathrm{A}, 1]$.

Suppose $C O N=\{A\}$ and $D E C=\{C\}$ hold. In this case $P O S_{\{A\}}(\{C\})=\{1,3\} \neq O B$, and $\{C\}$ does not depend on $\{A\}$. However, two implications from object 1 and 3 are seen as rules under the constraint that support $\geq 0.25$, accuracy $\geq 1.0$ and coverage $\geq$ 1.0. Since accuracy $=0.5$ holds for other implications, each other implication is not seen as a rule under this constraint.

\subsection{A Definition of Non-deterministic Information Systems}

A Non-deterministic Information System $(N I S)$ is also a quadruplet $(O B, A T$, $\left.\left\{V A L_{a} \mid a \in A T\right\}, g\right)$, where $g$ is a mapping from $O B \times A T$ to a power set of $\cup_{a \in A T} V A L_{a}$, i.e., $g: O B \times A T \rightarrow P\left(\cup_{a \in A T} V A L_{a}\right)$ (Orlowska and Pawlak, 1984; Orlowska, 1998). $g(x, a)$ is interpreted as if there is an actual value in this set but it is not known. This is the unknown interpretation for incomplete information (Lipski, 1981; Grzymala-Busse, 1991; Kryszkiewicz, 1998). Especially if the actual value is not known at all, $g(x, a)$ is equal to $V A L_{a}$. This is the same as null value interpretation (Codd, 1970).

\section{Rules in Non-deterministic Information Systems}

This section defines possible implications in NISs and two kinds of constraints for them. A rule is defined by a possible implication satisfying two kinds of constraints.

\subsection{Possible Implications in Non-deterministic Information Systems}

An example of a $N I S$ clarifies problems in $N I S s$, and possible implications in $N I S s$ are proposed.

EXAMPLE 3.1. Let us consider a $N I S$ such that $O B=\{1, \cdots, 10\}, A T=\{A, B, C, D\}$, $V A L_{1}=\{0,1,2\}, V A L_{2}=\{0,1,2,3\}, V A L_{3}=V A L_{4}=\{0,1,2,3,4,5\}$. Table 2 shows the function $g$ in $N I S$. In such a table, how do we deal with the dependency among attributes and rule extraction?

\begin{tabular}{|c|c|c|c|c|}
\hline$O B$ & $A$ & $B$ & $C$ & $D$ \\
\hline 1 & 0 & $\{2,3\}$ & 5 & 3 \\
\hline 2 & 0 & $\{0,1,3\}$ & 4 & 5 \\
\hline 3 & 2 & 3 & 5 & $\{0,4,5\}$ \\
\hline 4 & $\{0,1,2\}$ & 3 & 1 & 1 \\
\hline 5 & 1 & $\{0,1\}$ & 5 & 0 \\
\hline 6 & 2 & 1 & $\{3,5\}$ & 4 \\
\hline 7 & 1 & 0 & 2 & 2 \\
\hline 8 & 1 & 3 & $\{1,2,4\}$ & 4 \\
\hline 9 & 0 & 1 & 5 & 4 \\
\hline 10 & 2 & 1 & 0 & 1 \\
\hline
\end{tabular}

Table 2: A non-deterministic information system 
Definition 3.2. Let $N I S=\left(O B, A T,\left\{V A L_{a} \mid a \in A T\right\}, g\right)$ hold and $A T R$ be a set $\left\{a_{1}, \cdots, a_{n}\right\} \subset A T$. For any $x \in O B, P T(x, A T R)$ denotes Cartesian product $g\left(x, a_{1}\right) \times$ $\cdots \times g\left(x, a_{n}\right)$. We call an element a possible tuple (for ATR) of $x$. Every possible tuple $\zeta=\left(\eta_{a_{1}}, \cdots, \eta_{a_{n}}\right) \in P T(x, A T R)$ is identified with a formula $\bigwedge_{1 \leq i \leq n}\left[a_{i}, \eta_{a_{i}}\right]$.

Definition 3.3. For any $N I S$, let $C O N$ be condition attributes and $D E C$ be decision attributes. For any $x \in O B, I M P(x, C O N, D E C)$ denotes a set $\{\zeta \Rightarrow \eta \mid \zeta \in$ $P T(x, C O N), \eta \in P T(x, D E C)\}$. We call an element $\zeta \Rightarrow \eta$ a possible implication from $x$.

Let us consider a case that $C O N=\{A, B, C\}$ and $D E C=\{D\}$ in Example 3.1. $P T(1,\{A, B, C\})=\{(0,2,5),(0,3,5)\}, P T(1,\{D\})=\{(3)\}$ and $I M P(1,\{A, B, C\},\{D\})=$ $\{[A, 0] \wedge[B, 2] \wedge[C, 5] \Rightarrow[D, 3],[A, 0] \wedge[B, 3] \wedge[C, 5] \Rightarrow[D, 3]\}$ hold. A rule in a $N I S$ is defined by a possible implication satisfying some kinds of constraints. Some kinds of constraints to possible implications characterize rules in NISs. In the subsequent sections, two kinds of constraints are proposed in $N I S s$, and they are applied to possible implications.

\subsection{Constraint 1: Data Dependencies}

Data dependencies in DISs are extended to dependencies in NISs.

Definition 3.4. Let us consider a $N I S=\left(O B, A T,\left\{V A L_{a} \mid a \in A T\right\}, g\right)$, a set of attributes $A T R \subset A T$ and a mapping $h: O B \times A T R \rightarrow \cup_{a \in A T R} V A L_{a}$ such that $h(x, a) \in$ $g(x, a)$ for any $x \in O B$ and any $a \in A T R$. We call a $D I S=\left(O B, A T R,\left\{V A L_{a} \mid a \in\right.\right.$ $A T R\}, h$ ) a derived $D I S$ (for $A T R$ form $N I S$ ). We call an equivalence relation in a derived DIS a possible equivalence relation (pe-relation), and call an element in a perelation a possible equivalence class (pe-class).

In Table 2, there are $648\left(=2^{3} \times 3^{4}\right)$ derived $D I S s$ for $\{A, B, C, D\}$, and there are $6(=2 \times 3)$ derived $D I S s$ for $\{C\}$, According to the interpretation of $g(x, a)$, it is known that there is a derived $D I S$ with real information, but it is not known.

DEFINITION 3.5. Let us consider a $N I S$, condition attributes $C O N$, decision attributes $D E C$ and all derived $D I S_{1}, \cdots, D I S_{m}$ for $C O N \cup D E C$. For two threshold values $v a l_{1}$ and $v_{a l}\left(0 \leq v l_{1}, v_{2} \leq 1\right)$, if conditions 1 and 2 hold then we see $D E C$ depends on $C O N$ in $N I \bar{S}$.

1. $\mid\left\{D I S_{i} \mid D I S_{i}(1 \leq i \leq m)\right.$ is consistent $\} \mid / m \geq v a l_{1}$.

2. $\min _{i}\left\{\right.$ degree of dependency in $\left.D I S_{i}(1 \leq i \leq m)\right\} \geq v a l_{2}$.

In Definition 3.5, condition 1 requires most derived DISs are consistent, and condition 2 specifies the minimum value of degrees of dependency. This dependency is applied as a constraint to defining rules in NISs.

\subsection{Constraint 2: Six Groups of Possible Implications}

Six groups of possible implications are introduced in NISs. These groups are useful for characterizing each possible implication. 


\begin{tabular}{|c|c|c|c|}
\hline & Globally_consistent & Marginal & Globally_inconsistent \\
\hline Definite & $D G C$ & $D M$ & $D G I$ \\
\hline Indefinite & $I G C$ & $I M$ & $I G I$ \\
\hline
\end{tabular}

Table 3: Six groups of possible implications in NISs

DeFINITION 3.6. Let us consider a $N I S$, condition attributes $C O N$, decision attributes $D E C$ and any object $x \in O B$. For any possible implication $\psi \in I M P(x, C O N$, $D E C), D D I S(\psi, x, C O N, D E C)$ denotes a set $\{\phi \mid \phi$ is such a derived DIS for CON $U$ $D E C$ that an implication $d_{x}\left|C O N \Rightarrow d_{x}\right| D E C$ in $\phi$ is equal to $\left.\psi\right\}$.

Definition 3.7. Let us consider a $N I S$, condition attributes $C O N$, decision attributes $D E C$ and any object $x \in O B$. If $I M P(x, C O N, D E C)$ is a singleton set $\{\psi\}$, we say $\psi$ is definite. Otherwise we say each $\psi \in I M P(x, C O N, D E C)$ is indefinite. If $D D I S(\psi, x, C O N, D E C)=\{\phi \in D D I S(\psi, x, C O N, D E C) \mid x$ is consistent with other objects in $\phi\}$, we say $\psi$ is globally consistent. If $\{\phi \in D D I S(\psi, x, C O N, D E C) \mid x$ is consistent with other objects in $\phi\}=\emptyset$, we say $\psi$ is globally inconsistent. Otherwise we say $\psi$ is marginal. Finally, six groups in Table 3 are defined.

Let us consider a possible implication $\psi:[B, 1] \Rightarrow[C, 5] \in I M P(5,\{B\},\{C\})$ in Table 2. There exist $72\left(=2^{3} \times 3^{2}\right)$ derived $D I S s$ for $\{B, C\}$, and $D D I S(\psi, 5,\{B\},\{C\})$ consists of $36(=72 / 2)$ derived $D I S s$. Because object 5 is inconsistent with object 10 in all 36 derived $D I S s, \psi$ belongs to $I G I$ group. There exists no information incompleteness for each possible implication in definite groups. Possible implications in $D G C$ or $I G C$ groups are preferable, and possible implications in $D G I$ or $I G I$ groups are inappropriate. These six groups are also applied as another constraint to defining rules in NISs.

\subsection{A Definition of Rules in Non-deterministic Information Systems}

Definition 3.8. For any NIS, let $D E C$ be decision attributes. A rule (for $D E C$ ) from an object $x$ is a possible implication $\psi \in I M P(x, C O N, D E C)$ satisfying conditions below:

1. There exists a dependency from $C O N$ to $D E C$.

2. $\psi$ belongs to $D G C$ or $I G C$ groups in Table 3 .

Condition 1 follows data dependencies in DISs, and information incompleteness in $N I S s$ causes condition 2. Even though there may be other definitions of rules in NISs, rules in Definition 3.8 are dealt with in this paper.

In Definition 3.8 the most important problem is to find appropriate $C O N$ for specified $D E C$. In order to find appropriate $C O N$, it is necessary to know each degree of dependency in every derived $D I S$. For $C O N=\{A, B, C\}$ and $D E C=\{D\}$ in Example 3.1 , it is necessary to apply a procedure 648 times. The number of all derived DISs is equal to the number of products $\prod_{x \in O B, a \in C O N \cup D E C}|g(x, a)|$, and it increases in exponential order. Therefore a method, which sequentially applies the same procedure to all derived $D I S s$, is not appropriate for handling $N I S s$ with a large number of derived $D I S s$. For solving such a problem, we propose a method using pe-relations in NISs. Some other problems are also solved by applications of pe-relations. 


\section{A Method to Extract Rules in Non-deterministic Information Systems}

A method to extract rules (for specified decision attributes $D E C$ ) in any $N I S$ is proposed, which consists of seven steps.

(Step 1) Make a data file.

(Step 2) Execute a program transall for translating the data file to internal expressions in each attribute.

(Step 3) Execute a program pe to obtain all pe-relations in each attribute.

(Step 4) Execute a program merge to obtain all pe-relations for specified decision attributes $D E C$.

(Step 5) Fix condition attributes $C O N$, and execute a program merge to obtain all pe-relations for $C O N$.

(Step 6) Execute a program dependency to check the dependency from $C O N$ to $D E C$. If there exists a dependency, go to Step 7 else go to Step 5.

(Step 7) Execute a program extract to pick up possible implications belonging to $D G C$ or $I G C$ groups.

In order to clarify this method, a real execution handling Example 3.1 is presented. A workstation with $450 \mathrm{MHz}$ UltraSparc is employed for this experiment. The contents of data file data8.pl in Example 3.1 are as follows:

$\operatorname{object}(10,4)$. data $(1,[0,[2,3], 5,3])$. data $(2,[0,[0,1,3], 4,5]) . \cdots$

$\operatorname{data}(9,[0,1,5,4])$. $\operatorname{data}(10,[2,1,0,1])$.

The following is a real execution in Step 2.

?-transall.

File Name:'data8.pl'.

EXEC_TIME $=0.112(\mathrm{sec})$

yes

After this translation, four files A.rs, B.rs, C.rs and D.rs are produced. Step 3 is applied to these four files.

?-pe.

File Name: 'data8.pl'.

EXEC_TIME $=0.182(\mathrm{sec})$

yes

After this execution, four files A.pe, B.pe, C.pe and D.pe are produced. Every file stores all pe-relations in a format. In Step 4, suppose $D E C$ be $\{C\}$. All pe-relations for $\{C\}$ are stored in a file C.pe. In Step 5, all pe-relations for $\{A, B, D\}$ are produced by a program merge.

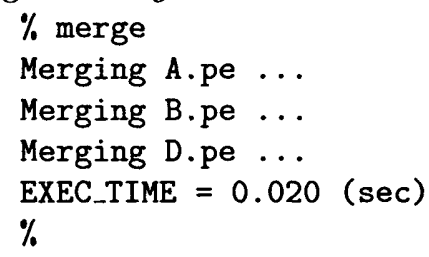


After this execution, a file ABD.pe is produced. It is known from this file that there exist 108 derived $D I S s$ for $\{A, B, D\}$, and there is only a kind of pe-relation, i.e., $\{\{1\},\{2\},\{3\},\{4\},\{5\},\{6\},\{7\},\{8\},\{9\},\{10\}\}$. Since any distinct objects $x, y \in O B$ are different each other, it is known every derived $D I S$ (for $C O N=\{A, B, D\}$ and $D E C=$ $\{C\}$ ) is consistent. A program dependency is executed in Step 6. Here, $v^{a l_{1}}$ and $\mathrm{val}_{2}$ in Definition 3.5 are fixed to 0.5 and 0.6 , respectively.

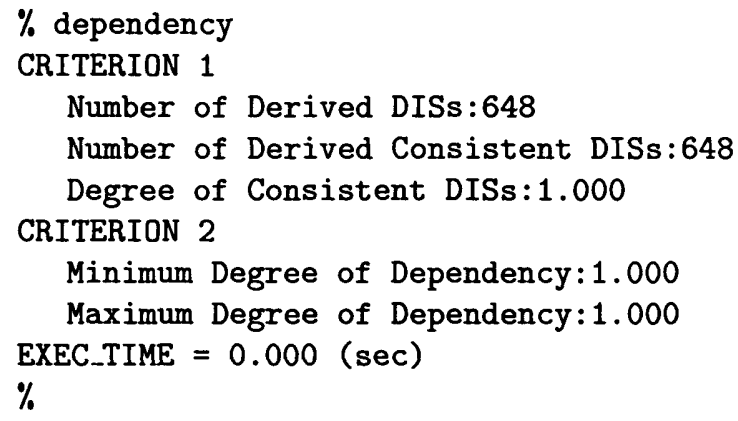

This execution shows a preferable result, namely each degree of dependency from $\{A, B$, $D\}$ to $\{C\}$ is 1 . By repeating Step 5 and Step 6, some kinds of dependencies are checked, and $C O N$ is fixed to a set $\{A, D\}$. In Step 7,CON=\{A,D\} and $D E C=\{C\}$ are specified in a file attrib.pl, and either a program extract or extractall is executed. Because there exists a dependency from $\{A, D\}$ to $\{C\}$, most possible implications belong to $D G C$ or $I G C$ groups.

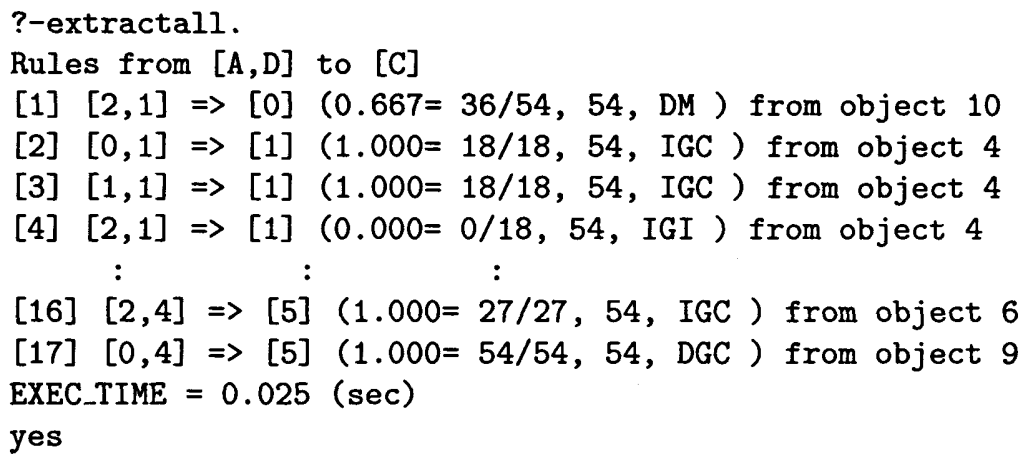

In this way it is possible to obtain rules in any NIS. Even though there exists work handling logic in NISs, there seems no work processing these rules in NISs. Of course, there exists a lot of work handling rules in $D I S s$.

In the subsequent sections algorithms of programs are examined. Every equivalence relation is effectively applied to extracting rules in any $D I S$, and every pe-relation is analogically applied to extracting rules in any NIS. Every program is realized according to pe-relations.

\section{Algorithms for Obtaining pe-relations}

In this section, algorithms in Step 2 and Step 3 are examined. An algorithm for solving the definability of a set in any NIS is first proposed, and an algorithm to obtain all pe-relations is also proposed. 


\subsection{An Algorithm for Solving the Definability of a Set in any NIS}

The definability of a set in DISs is extended to the definability of a set in NISs.

Definition 5.1. Let us consider a NIS and a set $X \subset O B . X$ is definable in $N I S$, if $X$ is definable in some derived $D I S s$ from $N I S$.

To check this, a method which sequentially examines the definability of a set for every derived DIS may be employed. However, this method will not be suitable for $N I S s$ with a large number of derived DISs. We propose another method according to Proposition 5.2.

Proposition 5.2. (Sakai, 2001b) Suppose there exist a $N I S$ and a set $X \subset O B$. $X$ is definable in NIS, if and only if there exist subsets $C L_{1}, \cdots, C L_{m}$ of $O B$, satisfying 1 and 2 below:

1. $\cup_{i} C L_{i}=X$,

2. $\left\{C L_{1}, \cdots, C L_{m}\right\}$ is a subset of a pe-relation.

According to this proposition, it is possible to check the definability of a set by finding $C L_{1}, \cdots, C L_{m}$. An algorithm in the following checks the definability of a set.

Algorithm 5.3. (Sakai, 2001b)

Input: A $N I S$ and a set $X \subset O B$.

Output: $X$ is definable in this $N I S$ or not.

(1) $X^{*}=X$ and $e q=\emptyset$.

(2) For any element $x \in X^{*}$, find a set $C L$ satisfying conditions (CL-1) and (CL-2). (CL-1) $x \in C L$ and $C L \subset X^{*}$,

$(\mathrm{CL}-2) e q \cup\{C L\}$ is a subset of a pe-relation.

$(2-1)$ If there is a set $C L$, then $[x]=C L, e q=e q \cup\{[x]\}$ and $X^{*}=X^{*}-C L$. If $X^{*} \neq \emptyset$ then go to (2). If $X^{*}=\emptyset$ then $X$ is definable.

(2-2) If there is no $C L$, then backtrack. If there is no branch for backtracking, then $X$ is not definable in this NIS.

This algorithm is similar to LEM1 and LEM2 algorithms (Grzymala-Busse and Werbrouck, 1998). The set $C L$ is unique in every $D I S$, but in NIS this set $C L$ may not be unique. Therefore, a search with backtracking is applied. At this point, Algorithm 5.3 is different from LEM1 and LEM2. In order to realize Algorithm 5.3, two extra properties of $p e$-relations are necessary (Sakai, 2001b).

\subsection{Basic Programs candidate and class}

A program class which checks the definability of a set in NIS is as follows: class (ATR, X) : - class0 (ATR, X, [],EQ, [], Pres, [] , Nres). class 0 (ATR, X, Y , EQ, Ppre, Pres, Npre, Nres)

$:-X==[], E Q=Y$, Pres $=$ Ppre, Nres $=$ Npre.

class 0 (ATR, [X|X1] , Y, EQ, Ppre, Pres, Npre, Nres)

:-candidate (ATR, [X|X1], CAN, Ppre, Pres1, Npre,Nres1),

minus ( $[X \mid X 1]$, CAN, REST), class0 (ATR, REST, [CAN|Y], EQ, Pres1, Pres, Nres1, Nres). 
A program candidate, which assigns a pe-class to the variable $C A N$, is realized due to the clarification of two properties for pe-relations. A program translate in Step 2 produces data for the program candidate. The following is a real execution for $A T R=\{A, B\}$, which is specified in a file attrib.pl.

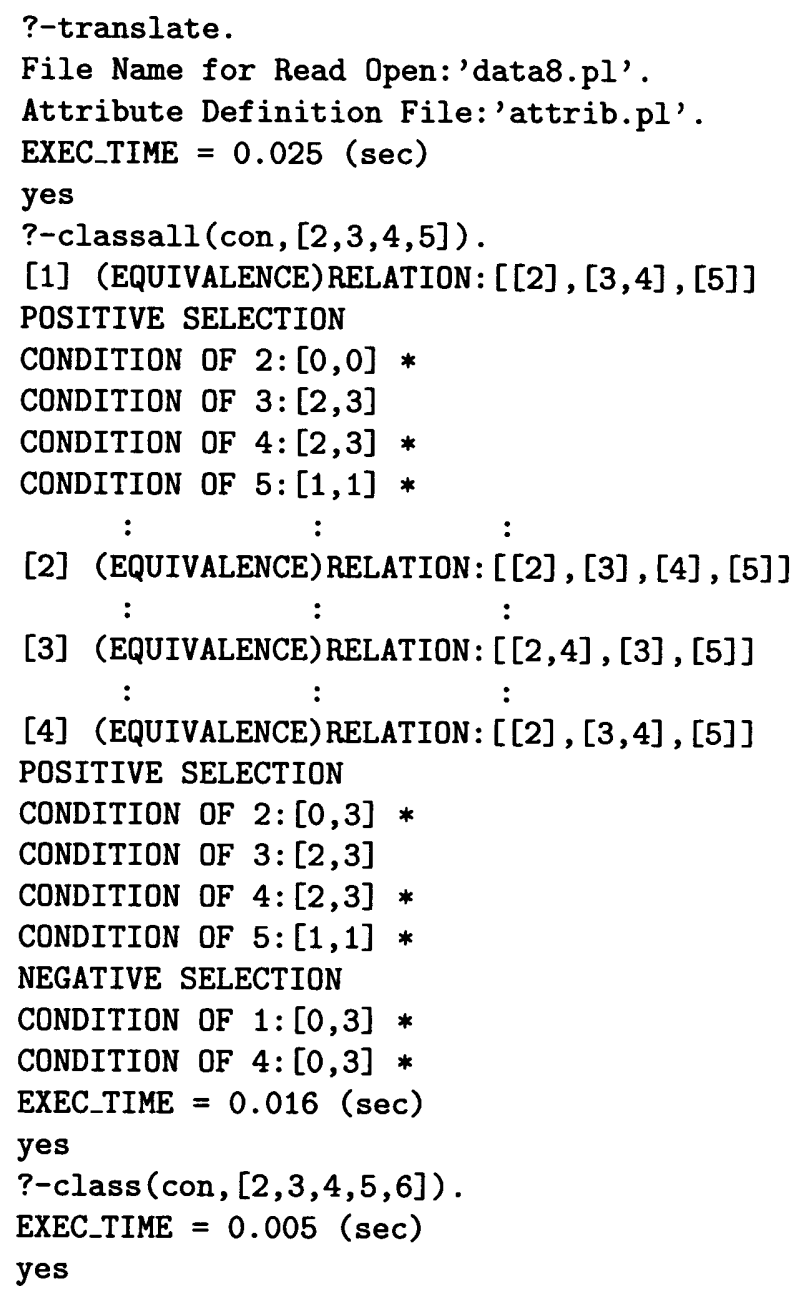

It is known that there are four kinds of relations which make a set $\{2,3,4,5\}$ definable. Every relation is stored in the variable $E Q$ in a clause class. A set $\{2,3,4,5,6\}$ is not definable in this $N I S$, because $[6]_{\{A, B\}}=\{6,10\}$ holds in any pe-relation.

\subsection{Side Effect of Algorithm 5.3}

Algorithm 5.3 has the following merit. After checking the definability of a set, a subset of a pe-relation is stored in the variable $E Q$ as a side effect of this algorithm. If $X=O B$, it is possible to obtain a pe-relation from this $E Q$. By applying this algorithm repeatedly until there is no branch for backtracking, all pe-relations can be obtained. 


\section{A Data Structure of pe-relations}

Usually, a number $i$ is identified with an object located in the $i$-th row of a table. By the order of row, it is possible to introduce the total order into $O B$. Suppose there exist $N$ kinds of pe-relations for $A T R$, and let peq $(A T R, k)$ denote the $k$-th $p e-$ relation for $A T R$. For handling $p e q(A T R, k)$, two arrays $h_{e a d}{ }_{A T R}[k][i]$ and $\operatorname{succ}_{A T R}[k][i]$ $(1 \leq k \leq N, 1 \leq i \leq|O B|)$ are employed. A set $[i]_{A T R, k} \in p e q(A T R, k)$ denotes a pe-class including object $i$. head $\operatorname{ATR}_{R}[k][j]$ for any $j \in[i]_{A T R, k}$ is the first element of the pe-class $[i]_{A T R, k}$, and $\operatorname{succ}_{A T R}[k][j]$ is the successor to $j$ in $[i]_{A T R, k}$. For the last element $j \in[i]_{A T R, k}, \operatorname{succ}_{A T R}[k][j]=0$. In such a data structure, every $p e$-class $[i]_{A T R, k}$ can easily be obtained. For every $i,[i]_{A T R, k}=\left\{\operatorname{head}_{A T R}[k][i], \operatorname{succ}_{A T R}[k][h e a d[k][i]], \cdots\right.$, $\operatorname{succ}_{A T R}[k]\left[\cdots \operatorname{succ}_{A T R}[k]\left[\right.\right.$ head $\left.\left.\left._{A T R}[k][i]\right]\right]\right\}$ holds.

For $A T R=\{A\}$ in Example 3.1, there exist three pe-relations, and $\operatorname{peq}(\{A\}, 3)=\{\{1$, $2,9\},\{3,6,10\},\{4,5,7,8\}\}$ is identified with two arrays below:

$\operatorname{head}_{\{A\}}[3][1]=1, \operatorname{succ}_{\{A\}}[3][1]=2, \operatorname{head}_{\{A\}}[3][2]=1, \operatorname{succ}_{\{A\}}[3][2]=9$,

$\operatorname{head}_{\{A\}}[3][3]=3, \operatorname{succ}_{\{A\}}[3][3]=6, \operatorname{head}_{\{A\}}[3][4]=4, \operatorname{succ}_{\{A\}}[3][4]=5$,

$\operatorname{head}_{\{A\}}[3][5]=4, \operatorname{succ}_{\{A\}}[3][5]=7, \cdots$, head $_{\{A\}}[3][10]=3, \operatorname{succ}_{\{A\}}[3][10]=0$.

For object 2 , head $\{A\}[3][2]=1, \operatorname{succ}_{\{A\}}[3][1]=2, \operatorname{succ}_{\{A\}}[3][2]=9$ and $\operatorname{succ}_{\{A\}}[3][9]=0$ hold, so it is known $[2]_{\{A\}, 3}=\{1,2,9\}$.

\section{An Algorithm for Checking Inclusion of pe-classes}

Let us show an important proposition.

Proposition 7.1. For a NIS and an $A T R \subset A T$, let us consider $t$-th pe-relation $\operatorname{peq}(A T R, t)$, i.e., two arrays head ${ }_{A T R}[t][i]$ and $\operatorname{succ}_{A T R}[t][i](1 \leq i \leq|O B|)$. For any $j \in O B, j \in[i]_{A T R, t}$ holds if and only if head $\operatorname{ATR}[t][j]=$ head $_{A T R}[t][i]$ holds.

Proof. If $j \in[i]_{A T R, t}$ holds, clearly head ${ }_{A T R}[t][j]=h e a d_{A T R}[t][i]$ holds. If $j \notin$ $[i]_{A T R, t}$ holds, $j$ belongs to other different class $\left[i^{\prime}\right]_{A T R, t}$. The first element of $[i]_{A T R, t}$ and the first element of $\left[i^{\prime}\right]_{A T R, t}$ are clearly different each other, and head ${ }_{A T R}[t][j] \neq$ $\operatorname{head}_{A T R}[t][i]$ is derived. Therefore if $\operatorname{head}_{A T R}[t][j]=h_{e a d}{ }_{A T R}[t][i]$ holds, $j \in[i]_{A T R, t}$ holds by contraposition.

By applying Proposition 7.1 to each $j \in[i]_{C O N, t}$, it is possible to check $[i]_{C O N, t} \subset$ $[i]_{D E C, s}$ or not. This inclusion implies every object in $[i]_{C O N, t}$ is consistent with other objects. Algorithm 7.2 solves this inclusion relation.

Algorithm 7.2. (Algorithm for checking the inclusion of pe-classes)

Input: Any object $i$, a pe-class $[i]_{C O N, t}$ for $C O N$ and a pe-class $[i]_{D E C, s}$ for $D E C$. Output: $[i]_{C O N, t} \subset[i]_{D E C, s}$ or not.

begin

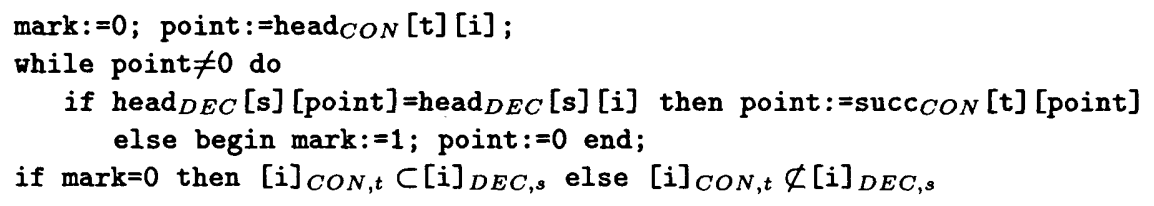
end.

Algorithm 7.2 sequentially picks up point $\in[i]_{C O N, t}$, and applies Proposition 7.1 to checking point $\in[i]_{D E C, s}$. The complexity of the worst case, which is a case such 
that $[i]_{C O N, t} \subset[i]_{D E C, s}$, is $o\left(\left|[i]_{C O N, t}\right|\right)$. Algorithm 7.2 is a basic algorithm for realizing a program dependency in Step 6.

\section{An Algorithm for Merging pe-relations}

A program merge in Step 5 is examined in this section.

Proposition 8.1. (Pawlak, 1991) Suppose $A, B \subset A T$ hold in a DIS. An equivalence relation $e q(A \cup B)$ is $\left\{M \subset O B \mid M=[i]_{A} \cap[i]_{B}\right.$ for $[i]_{A} \in e q(A)$ and $\left.[i]_{B} \in e q(B)(1 \leq i \leq|O B|)\right\}$.

Proposition 8.1 shows us a way to merge two equivalence relations. Algorithm 8.2 produces $\operatorname{peq}\left(A \cup B,,_{-}\right)$from $\operatorname{peq}(A, t)$ and $\operatorname{peq}(B, s)$.

Algorithm 8.2. (Sakai, 2001b)

Input: $\operatorname{peq}(A, t)$ for $A \subset A T$ and $p e q(B, s)$ for $B \subset A T$ respectively,

i.e., head ${ }_{A}[t][i], \operatorname{succ}_{A}[t][i]$, head $B[s][i]$ and $\operatorname{succ}_{B}[s][i](1 \leq i \leq|O B|)$.

Output: $p e q(A \cup B,-)$ for $A \cup B \subset A T$,

begin

i.e., head ${ }_{A \cup B}[-][i]$ and $\operatorname{succ}_{A \cup B}[-][i](1 \leq i \leq|O B|)$.

for $i:=1$ to $|O B|$ do begin head $A \cup B[][i]:=i ; \operatorname{succ}_{A \cup B}[][i]:=0$ end;

for $i:=1$ to $|O B|$ do if $\operatorname{head}_{A \cup B}[][i]=i$ then

begin

pre: $=i$; point: $=\operatorname{succ}_{A}[t][i]$;

while point $\neq 0$ do

begin if head $_{B}[\mathrm{~s}]\left[\right.$ point] $=\operatorname{head}_{B}[\mathrm{~s}][i]$ then

begin $\operatorname{succ}_{A \cup B}[][$ pre] : =point;

end;

$\operatorname{head}_{A \cup B}[][$ point $]:=i$; pre:=point

point : $=\operatorname{succ}_{A}[t][$ point $]$

end

end

end.

In Algorithm 8.2, if $\operatorname{peq}(A, t)=\{\{1,2, \cdots,|O B|\}\}$ and $\operatorname{peq}(B, s)=\{\{1\},\{2\}, \cdots$, $\{|O B|\}\}$, it is necessary to check head ${ }_{B}[s][$ point $]=$ head $d_{B}[s][i]$ for every point $\in[i]_{A, t}=$ $\{1,2, \cdots,|O B|\}$ and every $i \in O B$. Thus, computational complexity in the worst case is $o\left(|O B|^{2}\right)$. On the contrary, if $p e q(A, t) \subset \operatorname{peq}(B, s)$ holds, $[i]_{A, t} \subset[i]_{B, s}$ holds for any $i \in O B$. Therefore $\operatorname{head}_{B}[s][$ point $]=$ head $_{B}[s][i]$ always holds for any point $\in[i]_{A, t}$, and $\operatorname{head}_{B}[s][$ point $]=$ head $_{B}[s][i]$ is checked only once for any point $\in[i]_{A, t}$. Thus computational complexity in the best case is $o(|O B|)$.

Algorithm 8.2 is more effective for merging several kinds of attributes. Suppose there exists a sequence of attributes $A_{1}, A_{2}, \cdots, A_{m} \subset A T$, and it is necessary to obtain $e q\left(A_{1} \cup \cdots \cup A_{m}\right)$. In this case, Algorithm 8.2 is sequentially applied to $e q\left(A_{1} \cup \cdots \cup A_{k}\right)$ and $e q\left(A_{k+1}\right)(1 \leq k \leq m-1)$. Even though the order may be $o\left(|O B|^{2}\right)$ in the first application of Algorithm 8.2, the order is sequentially near to $o(|O B|)$. Because, every object is sequentially discerned by merging attributes.

\section{An Algorithm for Calculating the Dependencies}

A program dependency in Step 6 is examined in this section. Algorithm 9.1 calculates the degree of dependency by using pe-relations. 
Algorithm 9.1. (Algorithm for calculating the degree of dependency)

Input: $p e q(C O N, t)$ for attributes $C O N$ and $p e q(D E C, s)$ for attributes $D E C$.

Output: The degree of dependency based on $p e q(C O N, t)$ and $p e q(D E C, s)$.

begin

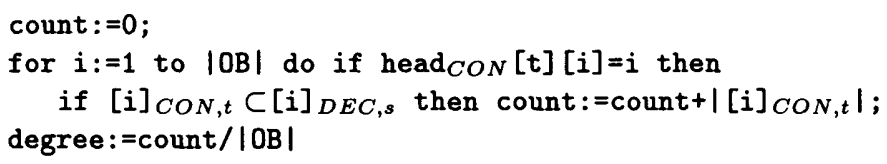

In Algorithm 9.1, inclusion $[i]_{C O N, t} \subset[i]_{D E C, s}$ is checked for any object $i$ satisfying head $_{C O N}[t][i]=i$. Since the order of checking this inclusion is less than $o\left(\left|[i]_{C O N, t}\right|\right)$, the order of Algorithm 9.1 is less than $o\left(\Sigma_{\left\{i \mid \text { head }_{C O N}[t][i]=i\right\}}\left|[i]_{C O N, t}\right|\right)$, which is equal to $o(|O B|)$. Because $[i]_{C O N, t}$ is an equivalence class, i.e., $\cup_{i \in O B}[i]_{C O N, t}=O B$ and $[i]_{C O N, t} \cap[j]_{C O N, t}=\emptyset$ for any two distinct classes hold.

Suppose there exist $N_{C O N}$ kinds of pe-relations for $C O N$ and $N_{D E C}$ kinds of perelations for $D E C$. By applying Algorithm 9.1 to these pairs of pe-relations $N_{C O N} \times$ $N_{D E C}$ times, it is possible to obtain each degree of dependency in all derived DISs. As stated before, the number of pairs of pe-relations are much smaller than the number of all derived $D I S s$. If $N_{C O N}=1$ and $N_{D E C}=1$, it is possible to check the dependency by only one time application of Algorithm 9.1 .

\section{Execution Time for Checking Dependencies}

In order to examine the execution time, four NISs in Table 4 are prepared, and each degree of dependency from $C O N=\{A, B, C\}$ to $D E C=\{D\}$ is calculated. Four $N I S s$ are produced by using a random number program.

\begin{tabular}{|c|c|c|c|c|}
\hline$N I S$ & $|O B|$ & $|A T|$ & $\left|V A L_{a}\right|(a \in A T)$ & Derived_DISs \\
\hline$N I S_{1}$ & 10 & 4 & 10 & 864 \\
\hline$N I S_{2}$ & 100 & 4 & 10 & 1944 \\
\hline$N I S_{3}$ & 300 & 4 & 10 & 3888 \\
\hline$N I S_{4}$ & 1000 & 4 & 10 & 7776 \\
\hline
\end{tabular}

Table 4: Definitions of NISs

Table 5 shows each execution time. The column of simple method shows expected values for obtaining each degree of dependency in all derived $D I S s$. An execution time for calculating the degree of dependency is measured in a derived $D I S$, and expected values

\begin{tabular}{|c|c|c|c|c|c||c|}
\hline$N I S$ & Step_2 & Step_3 & Step_5 & Step_6 & Total & simple_method \\
\hline$N I S_{1}$ & 0.087 & 0.241 & 0.000 & 0.000 & 0.328 & $0.000(=0.000 * 864)$ \\
\hline$N I S_{2}$ & 0.752 & 2.746 & 0.080 & 0.000 & 3.578 & $0.000(=0.000 * 1944)$ \\
\hline$N I S_{3}$ & 3.799 & 6.840 & 0.420 & 0.000 & 11.059 & $116.640(=0.030 * 3888)$ \\
\hline$N I S_{4}$ & 32.548 & 50.001 & 0.090 & 0.080 & 82.719 & $2643.840(=0.340 * 7776)$ \\
\hline
\end{tabular}

Table 5: Results of the execution time(sec) for dependency $[A, B, C] \Rightarrow[D]$ 


\begin{tabular}{|c|c|c|c|}
\hline$N I S$ & {$[A, D] \Rightarrow[B, C]$} & {$[A, C] \Rightarrow[B, D]$} & {$[D] \Rightarrow[A, B, C]$} \\
\hline$N I S_{1}$ & 0.000 & 0.000 & 0.000 \\
\hline$N I S_{2}$ & 0.600 & 0.040 & 0.070 \\
\hline$N I S_{3}$ & 0.380 & 0.440 & 0.420 \\
\hline$N I S_{4}$ & 0.520 & 0.480 & 0.150 \\
\hline
\end{tabular}

Table 6: Results of the execution time in Step $5+$ Step 6(sec) for every dependency

are defined by this execution time multiplied the number of derived DISs. According to Table 5, Step 5 and Step 6 take less execution time. Step 3, which obtains all pe-relations for each attribute, is the most time-consuming.

Table 6 shows each execution time in Step 5 and Step 6 for three cases. In order to find appropriate $C O N$ for specified $D E C$, it may be necessary to calculate some kinds of dependencies. Suppose $N$ kinds of dependencies are calculated in a NIS. Total execution time is [execution time of (Step $1+\operatorname{Step} 2+\operatorname{Step} 3+$ Step 4$)+N \times$ \{execution time of (Step $5+$ Step 6)\}]. Because, Step 5 and Step 6 take less execution time, it is convenient for finding dependencies in a NIS. If simple method is employed in this case, total execution time is $N \times($ the number of derived $D I S s) \times($ execution time of checking a dependency in a $D I S$ ). In this way simple method is not suitable for finding appropriate attributes $C O N$. Proposed method is more effective than simple method.

\section{Program extractall in Step 7}

A program extractall in Step 7 is examined in this section. This program is now implemented by prolog, and this program sequentially compares possible implications $\psi_{x} \in I M P(x, C O N, D E C)$ and $\psi_{y} \in I M P(y, C O N, D E C)$. The computational order is $o\left(\left(\sum_{1 \leq i \leq|O B|}|I M P(i, C O N, D E C)|\right)^{2}\right)$. This program took about $5(\mathrm{sec})$ in $N I S_{2}$, about 100 (sec) in $N I S_{3}$ and over 1000 (sec) in $N I S_{4}$ in Table 6. In this program, it is also necessary to apply pe-relations, however there is a problem. In Example 3.1 suppose $C O N=\{A, B\}$ and $D E C=\{D\}$ hold, and let us consider all pe-relations for $C O N$ and $D E C$. By using these pe-relations, it is easy to obtain the following response.

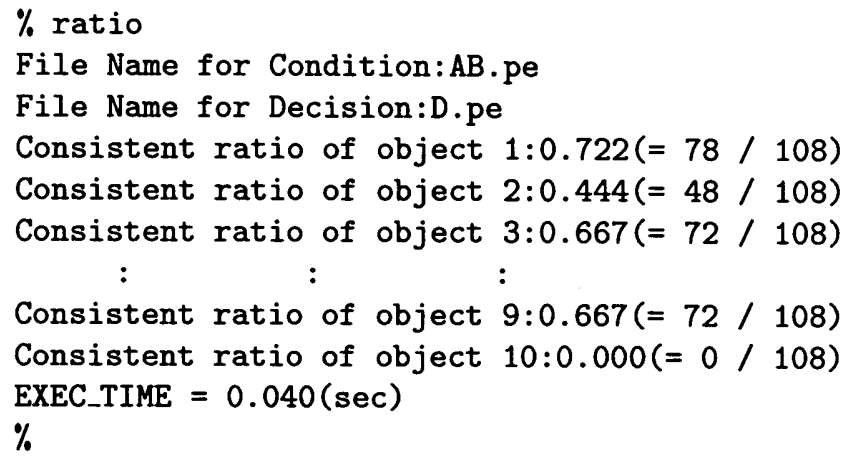

According to this response it is known object 1 is consistent with other objects in 78 derived DISs. This information is related to every object, and is not related to each possible implication. According to data structure of head $[*][*]$ and $\operatorname{succ}[*][*]$, it is possible to reach all pe-relations from any object. However, there is no relation between 
pe-relations and possible implications, and it is impossible to reach pe-relations from any possible implication. A new data structure connecting pe-relations and possible implications is necessary for improving the program extractall. It is also important to extend three criteria support $(\psi)$, accuracy $(\psi)$ and coverage $(\psi)$ in $D I S s$ to new criteria in NISs. An application of such criteria to rule extraction is under consideration.

\section{Concluding Remarks}

The rough sets based concept in DISs is extended to new concept in NISs for handling incomplete information, and a framework of rule extraction from non-deterministic information systems is proposed. Some algorithms using possible equivalence relations are also proposed for realizing programs. These programs may be useful tools for data analysis in non-deterministic information systems.

Up to now, there is only few work that deals with real data analysis in NISs. For handling not only certain information but also incomplete information, the research of rough sets based data analysis in NISs will be important. Furthermore, rough sets based data analysis in NISs could also be a mathematical foundation of knowledge discovery and data mining from incomplete information. Our work is toward real data analysis in NISs, and will extend the application area of rough sets theory.

\section{References}

Codd, E. (1970). A relational model of data for large shared data banks, Communication of the $A C M, 13,377-387$.

Grzymala-Busse, J. (1991). On the unknown attribute values in learning from examples, Lecture Notes in AI, Springer-Verlag, 542, 368-377.

Grzymala-Busse, J. (1997). A new version of the rule induction system LERS, Fundamenta Informaticae, IOS Press, 31, 27-39.

Grzymala-Busse, J. and Werbrouck, P. (1998). On the best search method in the LEM1 and LEM2 algorithms, Incomplete Information: Rough Set Analysis, Studies in Fuzziness and Soft Computing, Physica-Verlag, 13, 75-91.

Kryszkiewicz, M. (1998). Rough set approach to incomplete information systems, Information Sciences, 112, 39-49.

Kryszkiewicz, M. (1999). Rules in incomplete information systems, Information Sciences, 113, 271-292.

Lipski, W. (1981). On databases with incomplete information, Journal of the ACM, 28, 41-70.

Nakamura, A., Tsumoto, S., Tanaka, H. and Kobayashi, S. (1996). Rough set theory and its applications, Journal of Japanese Society for AI, 11, 209-215.

Orlowska, E. and Pawlak, Z. (1984). Representation of nondeterministic information, Theoretical Computer Science, 29, 27-39.

Orlowska, E. (1998). What you always wanted to know about rough sets, Incomplete Information: Rough Set Analysis, Studies in Fuzziness and Soft Computing, PhysicaVerlag, 13, 1-20. 
Pawlak, Z. (1982). Rough sets, Int'l. Journal of Information and Computer Sciences, 11, 341-356.

Pawlak, Z. (1991). Rough Sets, Kluwer Academic Publisher.

Pawlak, Z. (1996). Data versus logic a rough set view, Proc. 4th Int'l. Workshop on Rough Set, Fuzzy Sets and Machine Discovery, 1-8.

Polkowski, L. and Skowron, A.(Eds.) (1998a). Rough Sets in Knowledge Discovery 1, Studies in Fuzziness and Soft Computing, Physica-Verlag, 18.

Polkowski, L. and Skowron, A.(Eds.) (1998b). Rough Sets in Knowledge Discovery 2, Studies in Fuzziness and Soft Computing, Physica-Verlag, 19.

Ras, Z. and Joshi, S. (1997). Query approximate answering system for an incomplete DKBS, Fundamenta Informaticae, IOS Press, 30, 313-324.

Sakai, H. (1998). Some issues on nondeterministic knowledge bases with incomplete and selective information, Lecture Notes in AI, Springer-Verlag, 1424, 424-431.

Sakai, H. and Okuma, A. (1999). An algorithm for finding equivalence relations from tables with non-deterministic information, Lecture Notes in AI, Springer-Verlag, 1711, 64-72.

Sakai, H. and Okuma, A. (2000). An algorithm for checking dependencies of attributes in a table with non-deterministic information: a rough sets based approach, Lecture Notes in AI, Springer-Verlag, 1886, 219-229.

Sakai, H. (2001a). Two procedures for dependencies among attributes in a table with non-deterministic information: a summary, Lecture Notes in AI, Springer-Verlag, 2253, 301-305.

Sakai, H. (2001b). Effective procedures for handling possible equivalence relations in non-deterministic information systems, Fundamenta Informaticae, IOS Press, 48, 343-362.

Tsumoto, S. (2000). Knowledge discovery in clinical databases and evaluation of discovered knowledge in outpatient clinic, Information Sciences, 124, 125-137.

Zhong, N., Dong, J., Fujitsu, S. and Ohsuga, S. (1998). Soft techniques to rule discovery in data, Transactions of Information Processing Society of Japan, 39, 2581-2592.

Received March 16, 2001

Revised April 10, 2002 